Article

\title{
Chromatographic Separation on Synthetic Adsorbents
}

Kazuhiko TOKUNAGA, Shingo ANDOU, and Tadashi ADACHI

Separation Materials Laboratory, Specialty Chemicals Research Center, Mitsubishi Chemical Corporation, 1000 Kamoshida-cho, Aoba-ku, Yokohama, 227-8502, Japan

(Manuscript submitted February 15, 2003; accepted April 21,2003)

\begin{abstract}
Separation of Cephalosporin-C was investigated on synthetic adsorbents in a column liquid-chromatography to obtain fundamental data for industrial application. Two types of synthetic adsorbents with different porosity were tested, and their chromatographic behaviors were compared. The difference in the chromatographic peak sharpness and the peak separation can be explained by the difference in the porosity of the adsorbents.
\end{abstract}

\section{Introduction}

Synthetic adsorbents are insoluble three-dimensional crosslinked polymer beads with macropores.[1,2] They are widely used in liquid phase adsorption. Unlike other types of adsorbents such as activated carbon and zeolite, synthetic adsorbents show relatively mild adsorptivity and thus adsorbed compounds can be eluted under mild conditions. For this reason synthetic adsorbents have been applied to large scale separation of antibiotics such as Cephalosporin-C (Ceph-C) produced by fermentation. [3,4]

Adsorption and desorption phenomena on synthetic adsorbents are generally affected by their porosimetric parameters, such as specific surface area, pore volume, pore size, and pore size distribution. Earlier studies showed that the adsorption capacity for Ceph-C increases with increasing the specific surface area.[1,2] Porosity of dry state adsorbents can be measured by nitrogen adsorption / desorption technique. The porosity measured in dry state is considered to reflect the liquid-phase property of synthetic adsorbents.

High efficiency is an important requirement for industrial separation of Ceph-C. Model adsorption and desorption studies using Ceph-C and synthetic adsorbents were carried out by other investigators.[3-7] To perform efficient separation and purification, various synthetic adsorbents with different porosity have been developed and are commercially available.[1,2]

To obtain fundamental data on the Ceph-C separation for industrial application, a model chromatographic separation has been investigated using Ceph-C commercial reagent and two types of synthetic adsorbents; a solution of Ceph-C reagent was loaded onto a column of a synthetic adsorbent and then eluted from the adsorbent with an alkaline buffer, and the elution behavior of $\mathrm{Ceph}-\mathrm{C}$ and the impurities were monitored. Two prototype synthetic adsorbents with different porosity are compared in terms of the Ceph-C peak shape and the peak separation in the elution chromatograms. We discuss approaches for improving purity and recovery of eluted Ceph-C on the basis of the characteristics of the chromatograms. 


\section{Experimental}

\subsection{Materials}

Ceph-C was from Sigma and was used as received. A Ceph-C solution of ca. 10,000 ppm was prepared by dissolving Ceph-C into a $10 \mathrm{mmol} \mathrm{dm}^{-3}$ citric acid buffer ( $\mathrm{pH} 3$ ).

Prototype synthetic adsorbents 1 and 2 were from Mitsubishi Chemical Corporation (Tokyo, Japan). The properties are shown in Table 1. Specific surface area was determined by the BET nitrogen adsorption technique for the dry adsorbents. Pore volume was determined by nitrogen adsortption apparatus, and average pore radius was determined from the pore volume distribution. Bead sizes for both 1 and 2 are uniform. Consequently the effect of bead size on chromatography can be excluded in this study.

Table 1. Properties of the synthetic adsorbents used in this study

\begin{tabular}{llllll}
\hline Adsorbent & $\begin{array}{l}\text { Bead diameter } \\
(\mu \mathrm{m})\end{array}$ & $\begin{array}{l}\text { Uniformity } \\
\text { coefficient }\end{array}$ & $\begin{array}{l}\text { Specific pore volume } \\
\left(\mathrm{cm}^{3} / \mathrm{g} \text {-polymer }\right)\end{array}$ & $\begin{array}{c}\text { Specific surface area } \\
\left(\mathrm{m}^{2} / \mathrm{g} \text {-polymer }\right)\end{array}$ & $\begin{array}{l}\text { Pore radius } \\
(\mathrm{nm})\end{array}$ \\
\hline 1 & 400 & 1.05 & 1.7 & 900 & 8 \\
2 & 400 & 1.05 & 2.1 & 1200 & 9 \\
\hline
\end{tabular}

The synthetic adsorbents were pretreated before use as follows. Each adsorbent was immersed in $\mathrm{MeOH}$ for 12 hours for regeneration. The adsorbent was transferred into a column and washed with demineralized water ( 5 bed volume (BV)). Each pretreated synthetic adsorbent was then packed into a column ID $20 \times 500 \mathrm{~mm}$ and kept at $5^{\circ} \mathrm{C}$.

\subsection{Ceph-C Sorption}

The Ceph-C sorption procedure is listed in Table 2. The flow rate was controlled at space velocity $(\mathrm{SV})=1.5($ linear velocity $(\mathrm{LV})=0.75 \mathrm{~m} / \mathrm{h})$ for each run

Table 2. The Ceph-C sorption procedure

\begin{tabular}{llll}
\hline procedure & SV & Feed Solution & Concentration \\
\hline feed & 1.5 & Ceph-C & ca.10,000 ppm \\
rinse & 1.5 & water & \\
elute & 1.5 & NaHCO $_{3}$ & $0.2 \mathrm{~mol} \mathrm{dm}^{-3}$ \\
rinse & 1.5 & water & \\
\hline
\end{tabular}

The effluent was fractioned and analyzed by HPLC according to the literature method.[4] The concentration of eluted Ceph-C was calculated from the peak area detected by HPLC for each fraction.

\section{Results and Discussion}

The concentration of eluted Ceph-C for the adsorbents 1 and 2 are plotted against the effluent volume in Figs. 1 and 2, respectively. The impurity concentrations detected by HPLC were calculated in the similar way as Ceph-C. The sum of the impurity concentrations is plotted against the effluent volume in Figs. 1 and 2. 


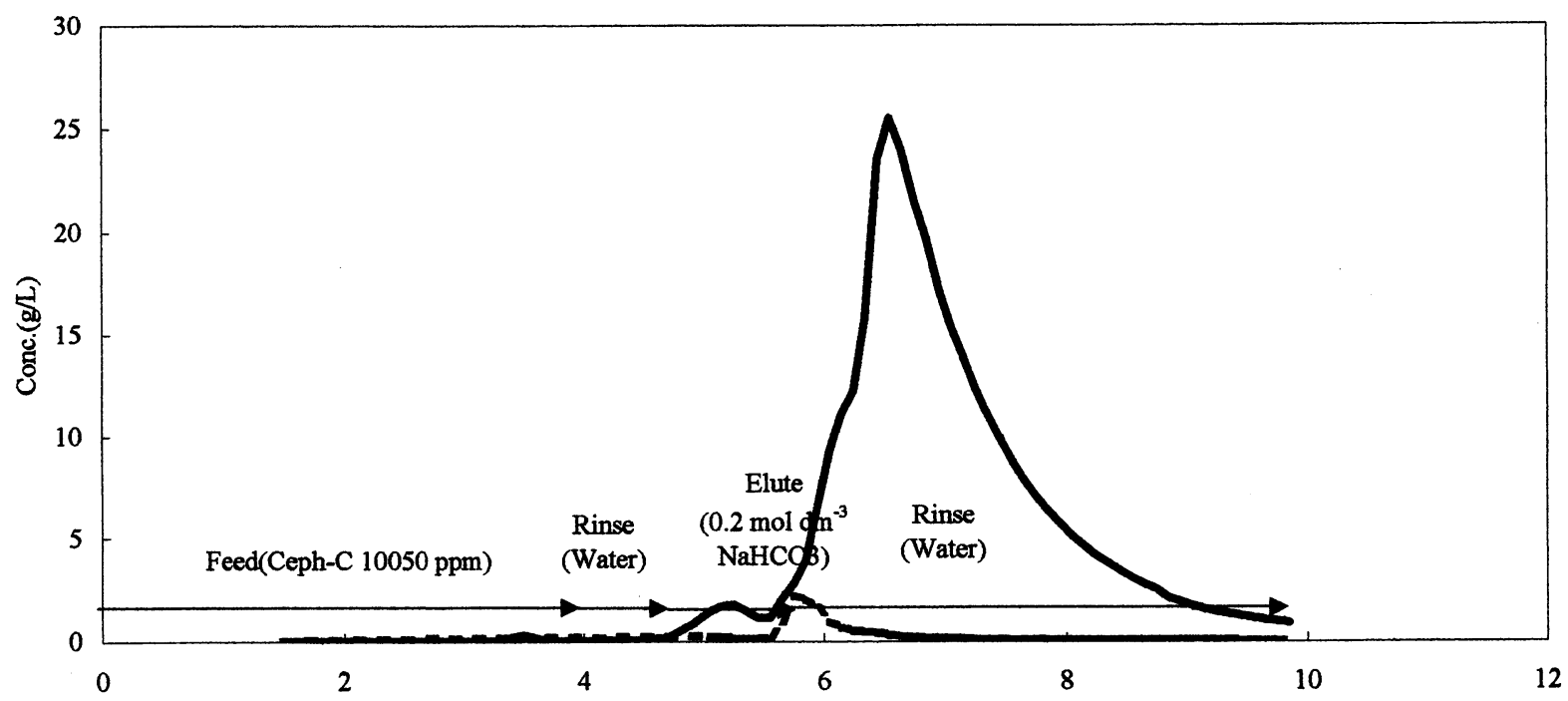

Fig 1. Concentration curves of eluted Ceph-C and the imprity vs elution volume (relative to bed volume) for the adsorbent $1\left(142 \mathrm{~cm}^{3}\right)$. Column ID $20 \times 500 \mathrm{~mm}$. Ceph-C; $10,050 \mathrm{ppm}$ in $10 \mathrm{mmol} \mathrm{dm}$ citric acid (pH 3). The solid line is calculated for Ceph-C, the broken line for the impurity.

The Ceph-C peak is sharper for the adsorbent 1 (Fig. 1), whereas the peak is broader for 2 (Fig. 2). The broader peak for 2 could be explained by greater Ceph-C adsorption for 2 . In fact the batch adsorption capacity for 1 is ca. $60 \mathrm{~g} / \mathrm{dm}^{3}$-polymer, and that for 2 is ca. $80 \mathrm{~g} / \mathrm{dm}^{3}$-polymer.[8] The elution of the greater amount of Ceph-C on 2 might take more time than the elution from 1 . The effect of greater specific surface area for 2 on the Ceph-C peak shape can also be assumed.

The peak sharpness for the adsorbent 1 could also be explained by the better size-exclusion effect for 1 . Possible explanation is that the adsorbent 1 can better exclude Ceph-C because the pore size of 1 is $1 \mathrm{~nm}$ smaller than 2 (Table 1).

The percentage of recovered Ceph-C from $6 \mathrm{BV}$ to $10 \mathrm{BV}$ relative to the initial Ceph-C load is estimated to be larger for 1 than that for 2. The elution of Ceph-C on 1 is almost completed at $10 \mathrm{BV}$ effluent volume (Fig. 1), whereas the Ceph-C elution still continue at $10 \mathrm{BV}$ for 2 (Fig. 2). The earlier elution and the better peak sharpness for 1 is considered to improve the recovery of Ceph-C. This suggests that the adsorbent 1 is more suitable for improvement of Ceph-C recovery.

The delayed elution of Ceph-C for the adsorbent 2 in comparison with that for 1 results in peak separation between Ceph-C and the impurity. The delay of Ceph-C elution for 2 relative to that for $\mathbf{1}$ is ca. $1 \mathrm{BV}$, whereas the retention time of the impurity was similar between 1 and 2. Consequently, the impurity peak for $\mathbf{2}$ is separated from the shoulder of the Ceph-C peak, while the impurity peak for 1 overlaps the Ceph-C elution. The greater adsorption capacity for 2 might delay the Ceph-C desorption. The better peak separation between Ceph-C and the impurity for $\mathbf{2}$ indicates that the adsorbent $\mathbf{2}$ is better suited for improvement of Ceph-C purity.

The results of the peak sharpness and the peak separation in Ceph-C elution suggest that the adsorption capacity and the porosity of the adsorbent affect the elution behavior. Both adsorbents have the different advantages, and this model chromatographic separation can be a tool for better choice of synthetic adsorbent. To better understand the factors for an efficient separation and purification, chromatographic study of various synthetic adsorbents are in due course. 


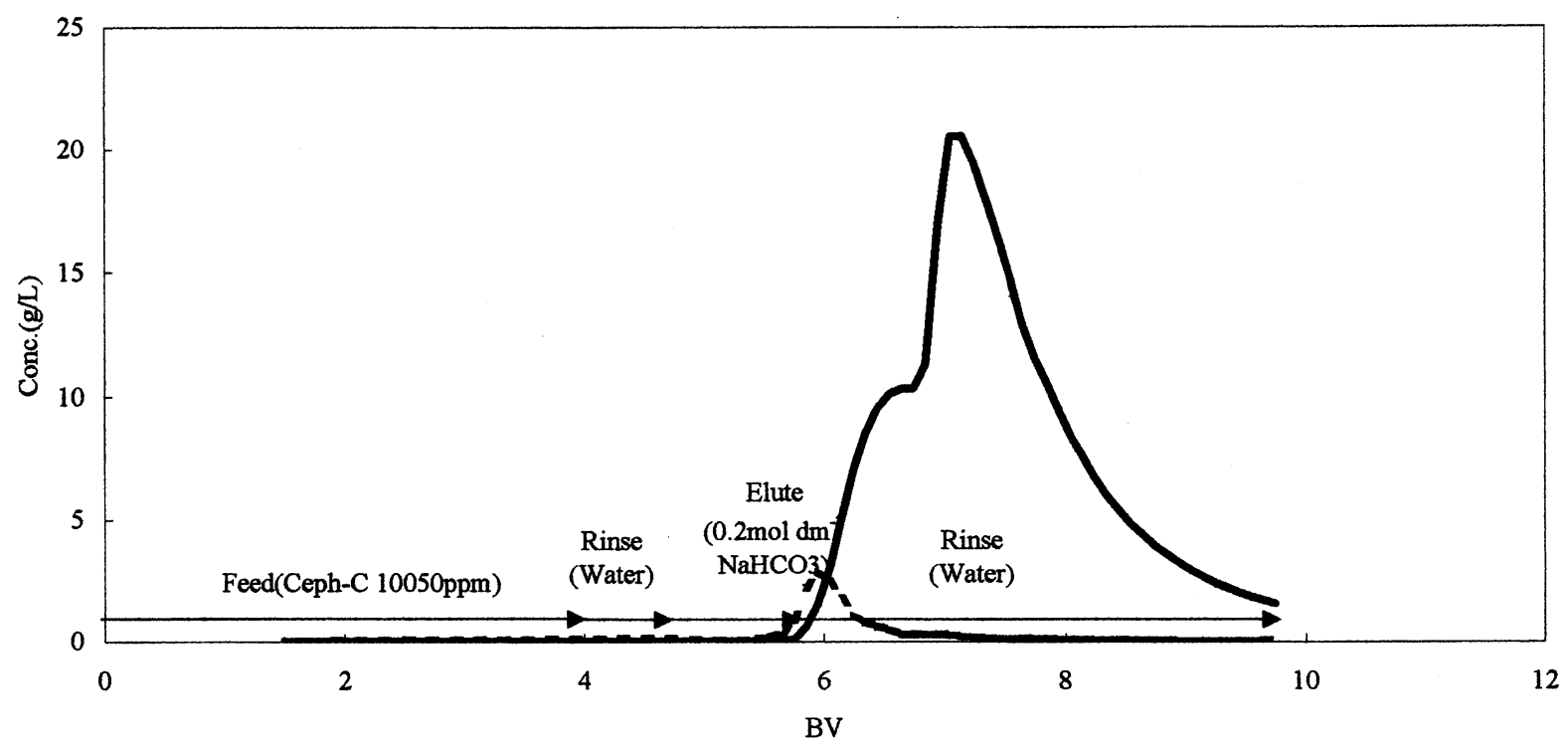

Fig. 2. Concentration curves of Ceph-C and the impurity vs elution volume (relative to bed volume) for the adsorbent $2\left(142 \mathrm{~cm}^{3}\right)$. Column ID $20 \times 500 \mathrm{~mm}$. Ceph-C; $10,050 \mathrm{ppm}$ in $10 \mathrm{mmol} \mathrm{dm}{ }^{-3}$ citric acid (pH 3). The solid line is calculated for Ceph-C, the broken line for the impurity.

\section{References}

1) "Diaion: Manual of Ion Exchange and Synthetic Adsorbent, Parts I and II," Mitsubishi Chemical Corporation (1992).

2) H. Takayanagi, J. Fukuda, and E. Miyata, "Downstream Processing of Natural Products" (Ed. M.S. Verrall), John Wiley \& Sons Ltd (1992), Chap. 11, pp 159-178.

3) W. Voser, J. Chem. Tech. Biotechnol., 32, 109-118 (1982).

4) Y. Xie, E. V. Sandt, T. Weerd, N. H. L. Wang, J. Chromatogr. A, 908, 273-291 (2001).

5) J. W. Lee, H. C. Park, and H. Moon, Separation and Purification Technology, 12, 1-11 (1997).

6) J. W. Lee, H. J. Jung, and H. Moon, Korean J. of Chem. Eng., 14, 277-284 (1997).

7) E. Firouztale, J. J. Maikner, K. C. Deissler, and P. G. Cartier, J. Chromatogr. A, 658, 361-370 (1994).

8) Unpublished result. 